\title{
Acoustic pressure pulses from laser-irradiated suspensions containing gold nanospheres in water: Experimental and theoretical study
}

\section{$\operatorname{AUTHOR}(\mathrm{S})$ :}

Shinto, Hiroyuki; Fukasawa, Tomonori; Aoki, Hiroyuki; Ito, Shinzaburo; Ohshima, Masahiro

\section{CITATION:}

Shinto, Hiroyuki ...[et al]. Acoustic pressure pulses from laser-irradiated suspensions containing gold nanospheres in water: Experimental and theoretical study. Colloids and Surfaces A: Physicochemical and Engineering Aspects 2013, 430: 51-57

\section{ISSUE DATE:}

2013-08

\section{URL:}

http://hdl.handle.net/2433/174320

\section{RIGHT:}

(C) 2013 Elsevier B.V.; This is not the published version. Please cite only the published version.; この論文は出版社版でありません。引用の際に は出版社版をご確認ご利用ください。 


\section{Acoustic pressure pulses from laser-irradiated suspensions containing gold nanospheres in water: Experimental and theoretical study}

Hiroyuki Shinto $^{\mathrm{a},{ }^{*}}$, Tomonori Fukasawa ${ }^{\mathrm{a}}$, Hiroyuki Aoki ${ }^{\mathrm{b}, \mathrm{c}}$, Shinzaburo Ito ${ }^{\mathrm{b}, \mathrm{c}}$, Masahiro Ohshima $^{\mathrm{a}}$

${ }^{a}$ Department of Chemical Engineering, ${ }^{b}$ Department of Polymer Chemistry, and ${ }^{c}$ Advanced Biomedical Engineering Research Unit, Kyoto University, Katsura, Nishikyo-ku, Kyoto 615-8510, Japan

Phone: +81-75-383-2671, Fax: +81-75-383-2651, e-mail: shinto@cheme.kyoto-u.ac.jp 
Abstract

We present a physical model that describes the acoustic pressure pulses from diluted suspensions of metal nanospheres in a liquid medium irradiated by laser pulses. The experimental measurements of the photoacoustic signals are also reported, where the suspensions of 8-nm gold nanospheres in water at different concentrations and different temperatures were irradiated by 0.8 -ns laser pulses. We mainly investigate how the nanosphere concentration and the suspension temperature influence the photoacoustic responses. The estimations from our model are found to be in good agreement with the results from the experiments.

Keywords:

Photoacoustic imaging; Photoacoustic pressure pulses; Nanosecond laser pulses; Contrast agents; Metal nanoparticles; Physical modeling. 


\section{Introduction}

Photoacoustic imaging (PAI) is a nonionizing and noninvasive imaging modality and is emerging as a novel technique for diagnostic imaging of various types of cancer [1-3]. When the tissue under inspection is illuminated by short laser pulses, the light energy is absorbed by the tissue to be converted to a thermoacoustic wave that outgoes from the illuminated region toward an acoustic transducer or an array of the transducers. The detected waves are used for reconstruction of the images of the tissue. The contrast in PAI relies on the optical-to-acoustic conversion (optoacoustic) efficiency, and can be exogenously improved by the use of nanoparticles (NPs) as contrast agents [2-6]. These NPs have optical absorption cross-sections much larger than those of tissue components, and are frequently used for enhancing the optical absorption of NP-labeled cells and tissues.

In the last decade, a great variety of NPs have been synthesized for the PAI contrast agents, such as gold NPs of various sizes and morphologies (e.g., spheres [7-11], rods [9,12,13], cages [14,15], and stars [16]), silica-coated gold nanospheres [17] and nanorods [18,19], gold-coated silica spheres [9,20,21], carbon nanotubes [22-24] and those covered with gold [25], dye-doped NPs [26,27], and nanocomposites with complex intrastructures [28-30]. Some of these NPs have been applied for the biomedical purposes, such as in vivo imaging in animals, selective detection of cancer, and imageguided therapy of cancer [7-16,20-26]. Besides the syntheses and biomedical applications of these contrast agents, however, there is so little known about the fundamental aspects of the photoacoustic phenomenon.

A recent study [17] has demonstrated that the acoustic signal generated from laser-irradiated suspensions of silica-coated gold nanospheres is influenced by the temperature- and material-dependent properties of the host liquids (i.e., water, toluene, and silicone oil) as well as by the thickness of the silica shell, and that the photoacoustic signal is dominated by the surrounding liquid rather than the nanospheres although the light absorbance of the former is still weaker than that of the latter. Several studies have estimated the temperature rise of a single nanosphere and its surrounding liquid (e.g., Refs. 
[11,31-34]). Only a few studies [17,35-37] have reported physical modeling of the acoustic signal produced from such a heated inhomogeneous solution including NPs (other than the particles of the larger sizes ranging from micrometers to millimeters) in the case of low-level laser irradiation. Nevertheless, the effects of NPs on the photoacoustic phenomena are not understood well and remain to be explored experimentally and theoretically from a fundamental point of view.

In the present study, we theoretically describe the acoustic pressure pulses from the heated suspensions containing spherical metal NPs in the case of low-level laser irradiation. We also report the experimental measurements of the photoacoustic signals, where the suspensions of 8-nm spherical gold NPs in water at different concentrations and different temperatures are irradiated by nanosecond laser pulses. We mainly investigate how the particle concentration and the suspension temperature influence the photoacoustic responses. The estimations from our model are compared with the results from the experiments.

\section{Experimental methods}

\subsection{Gold nanospheres}

A suspension of gold nanospheres in water (mean diameter $8.0 \mathrm{~nm}$, number concentration $5.70 \times 10^{12}$ spheres/mL; EM. GC10, Batch No. 14170) was purchased from British BioCell International (Cardiff, UK). The concentrated suspensions were prepared in clean test tubes by solvent evaporation using an aluminum block bath (DTU-2B; TAITEC, Saitama, Japan) at $60{ }^{\circ} \mathrm{C}$. The absorption spectra of these gold-nanosphere suspensions were measured before/after the concentration process, using Infinite M200 (Tecan, Grödig, Austria). No significant change was observed in the profile of the spectra and the wavelength for maximum absorption, indicating that the concentration process hardly affected the morphology and the monodispersity of the gold nanospheres. 
(a)

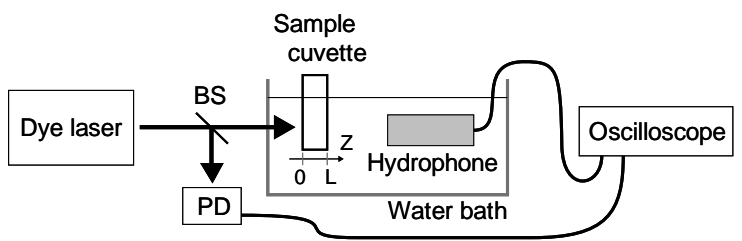

(b)

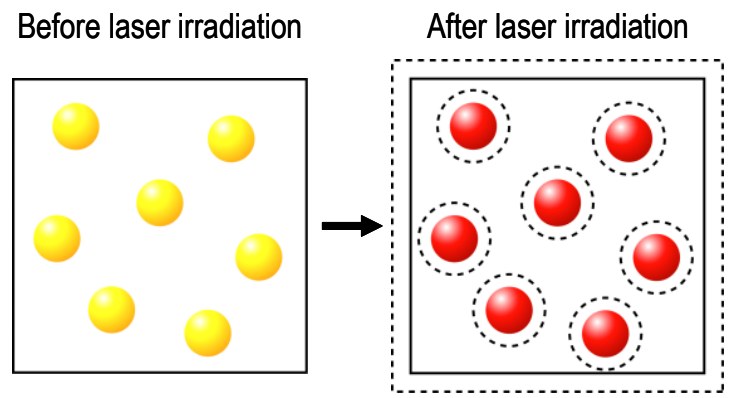

Fig. 1. (a) Illustration of the experimental setup. BS: beam splitter, PD: photo-diode. For more details, see the text. (b) Schematic representation of the monodispersed particle suspension before/after laser irradiation.

\subsection{Setup for photoacoustic signal detection}

Fig. 1a shows our experimental setup for photoacoustic signal detection. A sample cuvette was immersed in a temperature-controlled water bath and illuminated by pulses from a dye laser pumped by a nitrogen laser (OB-401/OB-4300; Optical Building Blocks, Birmingham, NJ, USA) at a wavelength of $509 \mathrm{~nm}$ and an energy of $107 \mu \mathrm{J} /$ pulse $\left(=I_{0}\right)$. The pulse width and repetition rate were $0.8 \mathrm{~ns}\left(=t_{\mathrm{L}}\right)$ and $5 \mathrm{~Hz}$, respectively. The beam diameter was $1 \mathrm{~mm}$, where the cross section of the specific region irradiated by the laser pulse was $s_{\mathrm{L}}=0.524 \mathrm{~mm}^{2}$ and the laser fluence was $F_{0} \equiv I_{0} / s_{\mathrm{L}}=204 \mathrm{~J} /\left(\mathrm{m}^{2}\right.$ pulse $)$. The acoustic signal from the laser-irradiated gold suspension was detected by a membrane-type hydrophone (H9C; Toray Engineering, Japan) with a measurable frequency range of 0.5-10 MHz. The distance between the hydrophone and the center of the sample cuvette was $\approx 38 \mathrm{~mm}$. The output signal from the hydrophone was recorded by an oscilloscope (TDS-2012; Tektronix, Beaverton, OR, USA) through an amplifier (Model 5682; Olympus NDT, Waltham, MA, USA). The split laser beam was 
detected by a photo-diode (DET10A; Thorlabs, Newton, NJ, USA) and fed to the oscilloscope as the trigger signal. The measurements were performed at the suspension temperatures of 4 and $20^{\circ} \mathrm{C}$, using different cuvettes of the light pass length of $L=0.92,4.2$, and $10.0 \mathrm{~mm}$. The results were obtained after averaging over 128 signals from every sample at each temperature. The absorption spectra of the goldnanosphere suspensions in these cuvettes were measured after the photoacoustic experiments. No significant change was observed in the profile of the spectra and the wavelength for maximum absorption, indicating that the laser irradiation hardly caused the fragmentation/aggregation of the gold nanospheres.

\section{Theoretical descriptions}

\subsection{Thermodynamic relation for a heated monodispersed suspension containing particles}

Fig. 1b depicts the monodispersed particle suspension before/after laser irradiation. Let us consider the case of low laser fluence, where the photoacoustic pressure observed from a laser-irradiated suspension containing NPs increases linearly with the laser fluence [31,38-42]. If the laser fluence exceeds a threshold, the photoacoustic pressure experiences a sharp nonlinear increase, where the water layer adjacent to heated metal NPs undergoes a phase transition from liquid to vapor $[31,32,38-48]$; this is out of our scope in the present study. It should be pointed out that the theoretical description given in this section is not limited to the spherical particles, but applicable to the particles with arbitrary shapes in principle.

The thermal expansion for a homogeneous medium of volume $V$ is described by

$$
\Delta V / V=-\kappa \Delta P+\beta \Delta T
$$

where $\Delta V, \Delta P$, and $\Delta T$ denote the changes in volume, pressure, and temperature, respectively; $\kappa$ is the isothermal compressibility and $\beta$ is the thermal coefficient of volume expansion. As shown in Fig. 1b, 
let us consider an inhomogeneous liquid containing particles, where heat evolution occurs both in the particles and in their surrounding liquid. In order to account for heat heterogeneity, we assume that a small volume element of the system, $V$, is divided into the fractional volumes of three regions: $V_{\mathrm{p}}$, the volume of the particles; $V_{\mathrm{m}-\mathrm{NF}}$, that of the medium next to the particles (near-field medium); and $V_{\mathrm{m}-\mathrm{FF}}$, that of the medium far from the particles (far-field medium). The temperature of the near-field medium is increased by laser irradiation, while that of the far-field medium remains at about the initial value. Hereafter, we assume that the physical properties of the particle and medium are constant at the initial temperature. For such a dispersion system, Eq. (1) should be modified as

$$
\begin{aligned}
\Delta V / V & =\phi_{\mathrm{p}}\left(\Delta V_{\mathrm{p}} / V_{\mathrm{p}}\right)+\phi_{\mathrm{m}-\mathrm{NF}}\left(\Delta V_{\mathrm{m}-\mathrm{NF}} / V_{\mathrm{m}-\mathrm{NF}}\right)+\phi_{\mathrm{m}-\mathrm{FF}}\left(\Delta V_{\mathrm{m}-\mathrm{FF}} / V_{\mathrm{m}-\mathrm{FF}}\right) \\
& =\phi_{\mathrm{p}}\left(-\kappa_{\mathrm{p}} \Delta P_{\mathrm{p}}+\beta_{\mathrm{p}} \Delta T_{\mathrm{p}}\right)+\phi_{\mathrm{m}-\mathrm{NF}}\left(-\kappa_{\mathrm{m}} \Delta P_{\mathrm{m}-\mathrm{NF}}+\beta_{\mathrm{m}} \Delta T_{\mathrm{m}-\mathrm{NF}}\right)+\phi_{\mathrm{m}-\mathrm{FF}}\left(-\kappa_{\mathrm{m}} \Delta P_{\mathrm{m}-\mathrm{FF}}+\beta_{\mathrm{m}} \Delta T_{\mathrm{m}-\mathrm{FF}}\right)
\end{aligned}
$$

where $V=V_{\mathrm{p}}+V_{\mathrm{m}-\mathrm{NF}}+V_{\mathrm{m}-\mathrm{FF}}, \Delta V=\Delta V_{\mathrm{p}}+\Delta V_{\mathrm{m}-\mathrm{NF}}+\Delta V_{\mathrm{m}-\mathrm{FF}}, \phi_{\mathrm{p}}+\phi_{\mathrm{m}-\mathrm{NF}}+\phi_{\mathrm{m}-\mathrm{FF}}=1$, and $\phi_{\mathrm{i}} \equiv V_{\mathrm{i}} / V(\mathrm{i}=\mathrm{p}$ $\mathrm{m}-\mathrm{NF}, \mathrm{m}-\mathrm{FF})$; especially, $\phi_{\mathrm{p}} \equiv n_{\mathrm{p}} v_{\mathrm{p}}$ is the volume fraction of the particles in the monodispersed suspension, where $v_{\mathrm{p}}$ is the volume of individual particles and $n_{\mathrm{p}}$ is the number of the particles per unit volume. The heat generated by the optical power deposition to every particle is defined as $e_{\text {tot }}$. Assuming that $\phi_{\mathrm{p}}$ is small so that every particle as well as its near-field medium are energetically independent of each other, the heat conduction from every particle to its surrounding medium results in the energy being remained inside the particle $\left(e_{\mathrm{p}}\right)$, that in the near-field medium $\left(e_{\mathrm{m}-\mathrm{NF}}\right)$, and that in the far-field medium $\left(e_{\mathrm{m}-\mathrm{FF}}\right)$, where $e_{\mathrm{tot}} \equiv e_{\mathrm{p}}+e_{\mathrm{m}-\mathrm{NF}}+e_{\mathrm{m}-\mathrm{FF}}$ : these fractional energies are a function of time during/after the laser irradiation. Then, the temperature rises of the particles $\left(\Delta T_{\mathrm{p}}\right)$, the near-field medium $\left(\Delta T_{\mathrm{m}-\mathrm{NF}}\right)$, and the far-field medium $\left(\Delta T_{\mathrm{m}-\mathrm{FF}}\right)$ are respectively given by

$$
\begin{aligned}
& \Delta T_{\mathrm{p}}=e_{\mathrm{p}} / c_{\mathrm{p}} \rho_{\mathrm{p}} v_{\mathrm{p}} \\
& \Delta T_{\mathrm{m}-\mathrm{NF}}=e_{\mathrm{m}-\mathrm{NF}}\left(n_{\mathrm{p}} V\right) / c_{\mathrm{m}} \rho_{\mathrm{m}}\left(\phi_{\mathrm{m}-\mathrm{NF}} V\right)=\left(\phi_{\mathrm{p}} / \phi_{\mathrm{m}-\mathrm{NF}}\right)\left(e_{\mathrm{m}-\mathrm{NF}} / c_{\mathrm{m}} \rho_{\mathrm{m}} v_{\mathrm{p}}\right) \\
& \Delta T_{\mathrm{m}-\mathrm{FF}}=\left(\phi_{\mathrm{p}} / \phi_{\mathrm{m}-\mathrm{FF}}\right)\left(e_{\mathrm{m}-\mathrm{FF}} / c_{\mathrm{m}} \rho_{\mathrm{m}} v_{\mathrm{p}}\right)
\end{aligned}
$$

where $c_{\mathrm{i}}$ and $\rho_{\mathrm{i}}(\mathrm{i}=\mathrm{p}, \mathrm{m})$ denote the specific heat capacity and the mass density, respectively. 
It is assumed that the laser pulse duration $t_{\mathrm{L}}$ is shorter than the acoustic confinement time, which is less than thermal confinement time [49]:

$$
t_{\mathrm{L}}<d_{\mathrm{c}} / C_{0, \mathrm{~m}}<d_{\mathrm{c}}^{2} / 4 \chi_{\mathrm{m}}
$$

where $d_{\mathrm{c}}$ is the characteristic length of heat heterogeneity, $C_{0, \mathrm{~m}}$ is the speed of sound wave in the medium, and $\chi_{\mathrm{m}}$ is the thermal diffusivity of the medium. For nanosecond laser pulses (i.e., $t_{\mathrm{L}} \sim 1 \mathrm{~ns}$ ), it is reasonable to assume that the fractional volume expansion is negligibly small (i.e., $\Delta V / V=0$ ), and that the pressures from the particles and their near-field medium relax much faster than that from the far-field medium (i.e., $\Delta P_{\mathrm{p}}=\Delta P_{\mathrm{m}-\mathrm{NF}}=0$ ). Under these assumptions, Eq. (2) with Eq. (3) results in

$$
\phi_{\mathrm{m}-\mathrm{FF}} \Delta P_{\mathrm{m}-\mathrm{FF}}=\left(n_{\mathrm{p}} / \kappa_{\mathrm{m}}\right)\left[\left(\beta_{\mathrm{p}} / c_{\mathrm{p}} \rho_{\mathrm{p}}\right) e_{\mathrm{p}}+\left(\beta_{\mathrm{m}} / c_{\mathrm{m}} \rho_{\mathrm{m}}\right) e_{\mathrm{m}}\right]
$$

where $e_{\mathrm{m}} \equiv e_{\mathrm{m}-\mathrm{NF}}+e_{\mathrm{m}-\mathrm{FF}}$. Consequently, the pressure pulse from the small volume element of interest $\left(p_{\text {tot }}\right)$ as well as two contributions from the particles $\left(p_{\mathrm{p}}\right)$ and the medium $\left(p_{\mathrm{m}}\right)$ are respectively given by

$$
\begin{aligned}
& p_{\text {tot }}=n_{\mathrm{p}} e_{\mathrm{tot}}\left[\left(\kappa_{\mathrm{p}} \Gamma_{\mathrm{p}} / \kappa_{\mathrm{m}}\right) \cdot x+\Gamma_{\mathrm{m}} \cdot(1-x)\right]=p_{\mathrm{p}}+p_{\mathrm{m}} \\
& p_{\mathrm{p}} \equiv\left(\kappa_{\mathrm{p}} \Gamma_{\mathrm{p}} / \kappa_{\mathrm{m}}\right) \cdot\left(n_{\mathrm{p}} e_{\mathrm{tot}}\right) x \\
& p_{\mathrm{m}} \equiv \Gamma_{\mathrm{m}} \cdot\left(n_{\mathrm{p}} e_{\mathrm{tot}}\right)(1-x)
\end{aligned}
$$

with

$$
\begin{aligned}
& \Gamma_{\mathrm{i}} \equiv \beta_{\mathrm{i}} / \kappa_{\mathrm{i}} c_{\mathrm{i}} \rho_{\mathrm{i}} \\
& x \equiv e_{\mathrm{p}} / e_{\mathrm{tot}}, \quad 1-x \equiv e_{\mathrm{m}} / e_{\mathrm{tot}}
\end{aligned}
$$

where $\Gamma_{\mathrm{i}}$ denotes the Grüneisen parameter for $\mathrm{i}(=\mathrm{p}, \mathrm{m})$ and $n_{\mathrm{p}} e_{\text {tot }}$ represents the volumetric optical absorption by the particle suspension. Equation (6) allows us to estimate the pressure pulse $p_{\text {tot }}$ as well as two contributions $p_{\mathrm{p}}$ and $p_{\mathrm{m}}$, after the thermal energies per particle, $e_{\mathrm{tot}}, e_{\mathrm{p}}$, and $e_{\mathrm{m}}$, are obtained.

\subsection{Heat production by a laser-irradiated metal nanosphere and the heat transfer into its surrounding} liquid 
For an analytical description of the thermal energies per particle without laser attenuation (i.e., $e_{\text {tot, } 0 \text {, }}$ $e_{\mathrm{p}, 0}$, and $\left.e_{\mathrm{m}, 0}\right)$, let us consider a single spherical metal particle of diameter $d_{\mathrm{p}}\left(=2 r_{\mathrm{p}}\right)$ suspended in an infinite liquid medium irradiated by a beam of pulsed laser light. Here, only the equations required for description of these thermal energies are explained, because their detailed derivation on the basis of Ref. [32] will be given elsewhere. As shown in Fig. 2a, the intensity of this light, $q(t)$, can be reasonably expressed by a triangular profile with the full width at half maximum (FWHM), $t_{\mathrm{L}}$, using

$$
q(t)= \begin{cases}q_{0}\left(t / t_{\mathrm{L}}\right), & 0 \leq t \leq t_{\mathrm{L}} \\ q_{0}\left(2-t / t_{\mathrm{L}}\right), & t_{\mathrm{L}} \leq t \leq 2 t_{\mathrm{L}} \\ 0, & t>2 t_{\mathrm{L}}\end{cases}
$$

where the laser fluence is given as $F_{0} \equiv q_{0} t_{\mathrm{L}}$.

We make the following assumptions: (i) the radiation energy absorbed by the nanosphere is homogeneously distributed over its volume, (ii) the spatial temperature inhomogeneity inside the nanosphere is negligibly small compared with the temperature distribution of the surrounding liquid medium, and (iii) no interfacial heat resistance exists between the nanosphere and its surrounding liquid medium. After analytical treatments of the heat transfer equations for the single nanosphere and surrounding medium with spherical symmetry, we obtain

$$
\begin{aligned}
& \Delta T_{\mathrm{p}}(t)=\left(\sigma_{\mathrm{abs}} q_{0} / 4 \pi k_{\mathrm{m}} r_{\mathrm{p}}\right)\left[J(t) \cdot U(t)-2 J\left(t-t_{\mathrm{L}}\right) \cdot U\left(t-t_{\mathrm{L}}\right)+J\left(t-2 t_{\mathrm{L}}\right) \cdot U\left(t-2 t_{\mathrm{L}}\right)\right], \quad r \leq r_{\mathrm{p}} \\
& \Delta T_{\mathrm{m}}(r, t)=\left[r_{\mathrm{p}}\left(r-r_{\mathrm{p}}\right) / 2 r \sqrt{\pi \chi_{\mathrm{m}}}\right] \int_{0}^{t}(t-\tau)^{-3 / 2} \exp \left[-\left(r-r_{\mathrm{p}}\right)^{2} / 4 \chi_{\mathrm{m}}(t-\tau)\right] \Delta T_{\mathrm{p}}(\tau) \mathrm{d} \tau, \quad r \geq r_{\mathrm{p}}
\end{aligned}
$$

with

$$
\begin{aligned}
& U(t)= \begin{cases}0, & t<0 \\
1, & t \geq 0\end{cases} \\
& J(t)=\left(\frac{\sqrt{\chi_{\mathrm{m}} t_{\mathrm{L}}}}{r_{\mathrm{p}}}\right)^{-2} \frac{\xi_{1} \xi_{2}}{\xi_{2}-\xi_{1}}\left\{\frac { 1 } { \xi _ { 1 } ^ { 3 } } \left[1-\frac{2}{\sqrt{\pi}}\left(\xi_{1} \frac{\sqrt{\chi_{\mathrm{m}} t}}{r_{\mathrm{p}}}\right)+\left(\xi_{1} \frac{\sqrt{\chi_{\mathrm{m}} t}}{r_{\mathrm{p}}}\right)^{2}-\operatorname{erfcx}\left(\xi_{1} \frac{\sqrt{\chi_{\mathrm{m}} t}}{r_{\mathrm{p}}}\right)_{]}-\right.\right. \\
& \frac{1}{\xi_{2}^{3}}\left[1-\frac{2}{\sqrt{\pi}}\left(\xi_{2} \frac{\sqrt{\chi_{\mathrm{m}} t}}{r_{\mathrm{p}}}\right)+\left(\xi_{2} \frac{\sqrt{\chi_{\mathrm{m}} t}}{r_{\mathrm{p}}}\right)^{2}-\left.\operatorname{erfcx}\left(\xi_{2} \frac{\sqrt{\chi_{\mathrm{m}} t}}{r_{\mathrm{p}}}\right)_{j}\right|_{j}\right.
\end{aligned}
$$



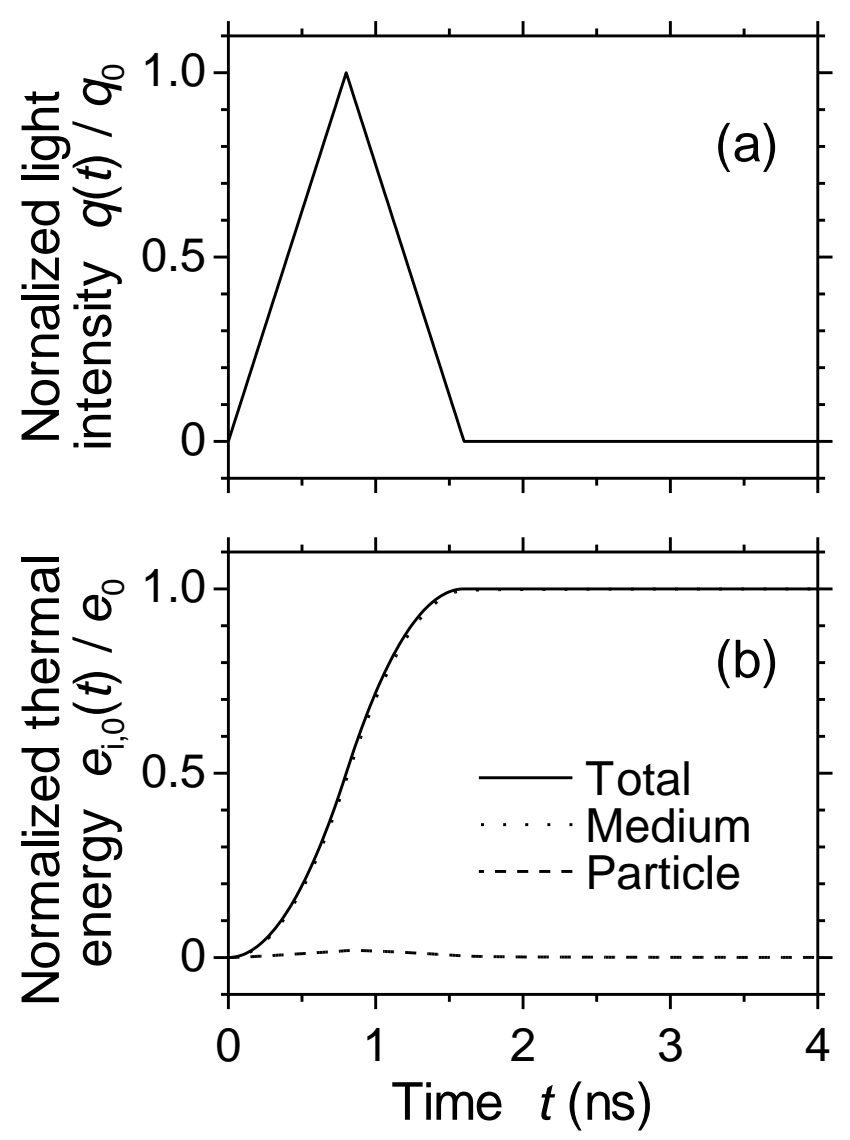

Fig. 2. (a) The normalized intensity of a laser beam with a triangular pulse of FWHM $t_{\mathrm{L}}=0.8 \mathrm{~ns}$, as a function of time $t$. (b) The total thermal energy produced by a laser-irradiated 8-nm gold sphere $\left[e_{\text {tot }, 0}(t)\right.$, solid line $]$, the thermal energy inside the nanosphere $\left[e_{\mathrm{p}, 0}(t)\right.$, long dashed line $]$, and the thermal energy of its surrounding water medium $\left[e_{\mathrm{m}, 0}(t)\right.$, short dashed line $]$, as a function of time $t$ at $20{ }^{\circ} \mathrm{C}$. Every energy $e_{\mathrm{i}, 0}(t)(\mathrm{i}=$ tot, $\mathrm{p}, \mathrm{m})$ was normalized by $e_{0} \equiv \sigma_{\mathrm{abs}} F_{0}$, which represents the optical power deposition per nanosphere.

$\xi_{1}, \xi_{2}=(3 / 2)[\alpha \mp \sqrt{\alpha(\alpha-4 / 3)}], \quad \alpha=c_{\mathrm{m}} \rho_{\mathrm{m}} / c_{\mathrm{p}} \rho_{\mathrm{p}}$

$\operatorname{erfcx}(x) \equiv \exp \left(x^{2}\right) \cdot \operatorname{erfc}(x)=\exp \left(x^{2}\right) \cdot[1-\operatorname{erf}(x)]$

where $\sigma_{\mathrm{abs}}$ is the absorption cross-section of the metal nanosphere, $k_{\mathrm{m}}$ is the thermal conductivity of the medium, and $\chi_{\mathrm{m}}\left(\equiv k_{\mathrm{m}} / c_{\mathrm{m}} \rho_{\mathrm{m}}\right)$ is the thermal diffusivity of the medium. 
In the absence of laser attenuation, the total thermal energy produced by a laser-irradiated particle $e_{\text {tot }, 0}(t)$, the thermal energy inside the nanosphere $e_{\mathrm{p}, 0}(t)$, and the thermal energy of its surrounding medium $e_{\mathrm{m}, 0}(t)$ are respectively given as a function of time:

$$
\begin{aligned}
e_{\mathrm{tot}, 0}(t) & =\sigma_{\mathrm{abs}} \int_{0}^{t} q(\tau) \mathrm{d} \tau=e_{0} \cdot f(t) \\
e_{\mathrm{p}, 0}(t) & =c_{\mathrm{p}} \rho_{\mathrm{p}} v_{\mathrm{p}} \Delta T_{\mathrm{p}}(t) \\
& =\left(e_{0} r_{\mathrm{p}}^{2} / 3 \alpha \chi_{\mathrm{m}} t_{\mathrm{L}}\right)\left\{J(t) \cdot U(t)-2 J\left(t-t_{\mathrm{L}}\right) \cdot U\left(t-t_{\mathrm{L}}\right)+J\left(t-2 t_{\mathrm{L}}\right) \cdot U\left(t-2 t_{\mathrm{L}}\right)\right\} \\
e_{\mathrm{m}, 0}(t) & =e_{0}\left[f(t)-\left(r_{\mathrm{p}}^{2} / 3 \alpha \chi_{\mathrm{m}} t_{\mathrm{L}}\right)\left\{J(t) \cdot U(t)-2 J\left(t-t_{\mathrm{L}}\right) \cdot U\left(t-t_{\mathrm{L}}\right)+J\left(t-2 t_{\mathrm{L}}\right) \cdot U\left(t-2 t_{\mathrm{L}}\right)\right\}\right]
\end{aligned}
$$

where

$$
\begin{aligned}
& e_{0} \equiv \sigma_{\text {abs }} F_{0}=\sigma_{\text {abs }} q_{0} t_{\mathrm{L}} \\
& f(t)= \begin{cases}\left(t / t_{\mathrm{L}}\right)^{2} / 2, & 0 \leq t \leq t_{\mathrm{L}} \\
-\left(t / t_{\mathrm{L}}\right)^{2} / 2+2\left(t / t_{\mathrm{L}}\right)-1, & t_{\mathrm{L}} \leq t \leq 2 t_{\mathrm{L}} \\
1, & t>2 t_{\mathrm{L}}\end{cases}
\end{aligned}
$$

The time variations of these energies, $e_{\mathrm{i}, 0}(t)(\mathrm{i}=\mathrm{tot}, \mathrm{p}, \mathrm{m})$, are shown in Fig. $2 \mathrm{~b}$ for a gold sphere of $d_{\mathrm{p}}$ $=8 \mathrm{~nm}$ in water irradiated by a triangular pulsed laser of $t_{\mathrm{L}}=0.8 \mathrm{~ns}$ at $20^{\circ} \mathrm{C}$, which corresponds to the experimental system mentioned in Section 2.2. It should be noted that we implicitly assumed no thermal expansion during/after laser irradiation in Fig. 2b as well as Eqs. (10), (11), and (16).

\subsection{Photoacoustic pressure pulse and effect of attenuation}

Once the time at an instant of thermal-to-acoustic conversion $\left(t_{\text {conv }}\right)$ is assumed properly, we can straightforward estimate the acoustic pressure pulse in the absence of laser and sound attenuation, using Eq. (6) with the values of $e_{\mathrm{i}, 0}(t)(\mathrm{i}=$ tot, $\mathrm{p}, \mathrm{m})$ at $t=t_{\text {conv }}$ that are obtained from Eq. (16) as shown in Fig. 2b. Following Egerev et al. [32], $t_{\text {conv }}$ was assumed to be the time for the end of laser irradiation, namely $t_{\text {conv }}=2 t_{\mathrm{L}}(=1.6 \mathrm{~ns})$ in the present study.

To take into account the effect of laser attenuation, we consider the system shown in Fig. 1a, where a 
monodispersed suspension of metal nanospheres in a cuvette of the light pass length of $L$ is irradiated by a laser pulse of the fluence of $F_{0}=q_{0} t_{\mathrm{L}}$. The local laser fluence and the local light intensity in this system are a function of the position $Z(=0-L)$ along the direction of laser irradiation:

$$
\begin{aligned}
& F(Z) \equiv F_{0} \cdot \exp \left(-n_{\mathrm{p}} \sigma_{\mathrm{ext}} Z\right) \\
& I(Z) \equiv I_{0} \cdot \exp \left(-n_{\mathrm{p}} \sigma_{\mathrm{ext}} Z\right)
\end{aligned}
$$

where $\sigma_{\text {ext }}$ is the extinction cross-section of the nanosphere and $I_{0} \equiv s_{\mathrm{L}} F_{0}$. In the present study, the optical absorbance $A_{\lambda}$ for the monodispersed suspension of the nanospheres in the cuvette of the light pass length of $L$ is defined by

$$
A_{\lambda} \equiv-\log _{10}\left[I(L) / I_{0}\right]=n_{\mathrm{p}} \sigma_{\mathrm{ext}} L / \log _{e} 10 \approx n_{\mathrm{p}} \sigma_{\mathrm{abs}} L / \log _{e} 10
$$

where we use Eq. (20) as well as $\sigma_{\text {ext }} \approx \sigma_{\text {abs }}$ that is valid for sufficiently small particles. Similar to Eqs. (19) and (20), the position-dependent energies of $e_{\mathrm{i}}(\mathrm{i}=\mathrm{tot}, \mathrm{p}, \mathrm{m})$ at $t=t_{\mathrm{conv}}\left(=2 t_{\mathrm{L}}\right)$ are represented as

$e_{\mathrm{i}}(Z)=\left.e_{\mathrm{i}, 0}\right|_{t=2 t_{\mathrm{L}}} \cdot \exp \left(-n_{\mathrm{p}} \sigma_{\mathrm{ext}} Z\right)$

Consequently, using Eqs. (6), (8), (21), and (22), the photoacoustic pressure pulses at positions $Z=0$ and $L, p_{\text {tot }}(0)$ and $p_{\text {tot }}(L)$, are given by

$$
\begin{aligned}
& p_{\text {tot }}(0)=n_{\mathrm{p}} e_{0} \cdot\left[\left(\kappa_{\mathrm{p}} \Gamma_{\mathrm{p}} / \kappa_{\mathrm{m}}\right) \cdot x+\Gamma_{\mathrm{m}} \cdot(1-x)\right] \approx\left[\left(F_{0} \log _{e} 10\right) / L\right]\left[\left(\kappa_{\mathrm{p}} \Gamma_{\mathrm{p}} / \kappa_{\mathrm{m}}\right) \cdot x+\Gamma_{\mathrm{m}} \cdot(1-x)\right] \cdot A_{\lambda} \\
& p_{\text {tot }}(L)=p_{\text {tot }}(0) \cdot \exp \left(-n_{\mathrm{p}} \sigma_{\text {ext }} L\right) \approx p_{\text {tot }}(0) \cdot 10^{-A_{\lambda}}
\end{aligned}
$$

with

$$
x \equiv e_{\mathrm{p}}(Z) / e_{\mathrm{tot}}(Z)=\left.\left(e_{\mathrm{p}, 0} / e_{\mathrm{tot}, 0}\right)\right|_{t=2 t_{\mathrm{L}}}=\left(r_{\mathrm{p}}^{2} / 3 \alpha \chi_{\mathrm{m}} t_{\mathrm{L}}\right)\left[J\left(2 t_{\mathrm{L}}\right)-2 J\left(t_{\mathrm{L}}\right)\right]
$$

where Eqs. (16)-(18) are also used.

In Section 4.2, we employed Eqs. (23)-(25) to estimate the photoacoustic pressure pulses for the experimental system depicted in Fig. 1a. The acoustic pressure generated inside the sample cuvette ranging from $Z=0$ to $L$ propagates to arrive at an observation point of the detector. Because the pressure pulse at $Z=L$ is closer to the observation point, it is first detected as $p_{1}^{\text {obs }}$. After the time 
period of $L / C_{0, \mathrm{~m}}$, the pressure pulse at $Z=0$ is detected as $p_{2}^{\text {obs }}$. The observed intensities of these two pressure pulses are estimated as

$$
\begin{aligned}
& p_{1}^{\mathrm{obs}}=C \cdot p_{\mathrm{tot}}(L)=C \cdot p_{\mathrm{tot}}(0) \cdot 10^{-A_{\lambda}} \\
& p_{2}^{\mathrm{obs}}=C \cdot p_{\text {tot }}(0) \cdot \exp \left(-\alpha_{\mathrm{s}} L\right)
\end{aligned}
$$

where $C$ is an apparatus constant. According to Chapter 4 of Ref. [50], the total attenuation of sound in the particle suspension, $\alpha_{\mathrm{s}}$, is expressed as $\alpha_{\mathrm{s}}=\alpha_{\mathrm{abs}}+\alpha_{\mathrm{sca}}+\alpha_{\mathrm{int}}$, where $\alpha_{\mathrm{abs}}$ and $\alpha_{\mathrm{sca}}$ are the attenuation due to the particle absorption and the particle scattering, respectively, and $\alpha_{\text {int }}$ is the background intrinsic attenuation due to the interaction with the materials of the particles and the medium, considered as homogeneous phases on a molecular level. In the case of small particles $\left(d_{\mathrm{p}}<100 \mathrm{~nm}\right)$ and/or low volume fractions ( $\left.\phi_{\mathrm{p}}<0.01\right), \alpha_{\mathrm{abs}} \approx 0$ and $\alpha_{\mathrm{sca}} \approx 0$, resulting in $\alpha_{\mathrm{s}} \approx \alpha_{\mathrm{int}} \approx \alpha_{\mathrm{m}}$ that denotes the intrinsic attenuation of the liquid medium and is independent of $d_{\mathrm{p}}$ and $\phi_{\mathrm{p}}$ (see Chapter 4 of Ref. [50]).

\section{Results and discussion}

\subsection{Observation of photoacoustic signals}

Fig. 3 displays the signal amplitudes observed from a laser-irradiated aqueous suspension of 8-nm gold nanospheres at $20^{\circ} \mathrm{C}$, as a function of time for different cuvettes of $L=0.92,4.2$, and $10.0 \mathrm{~mm}$. Two isolated spikes were observed: the positive-peak signals were first observed and followed by the negative-peak signals at the later time by $0.62,2.70$, and $6.66 \mu \mathrm{s}$ for $L=0.92,4.2$, and $10.0 \mathrm{~mm}$, respectively. The plane waves generated at the inner walls of the cuvette (i.e., $Z=0$ and $L$ ) propagated to arrive at an observation point of the detector. Because the plane wave at $Z=L$ was closer to the observation point, it should be first detected. The plane wave at $Z=0$ should be detected after the time period of $L / C_{0, \mathrm{~m}}=0.62,2.84$, and $6.75 \mu \mathrm{s}$ for $L=0.92,4.2$, and $10.0 \mathrm{~mm}$, respectively, where $C_{0, \mathrm{~m}}=$ 


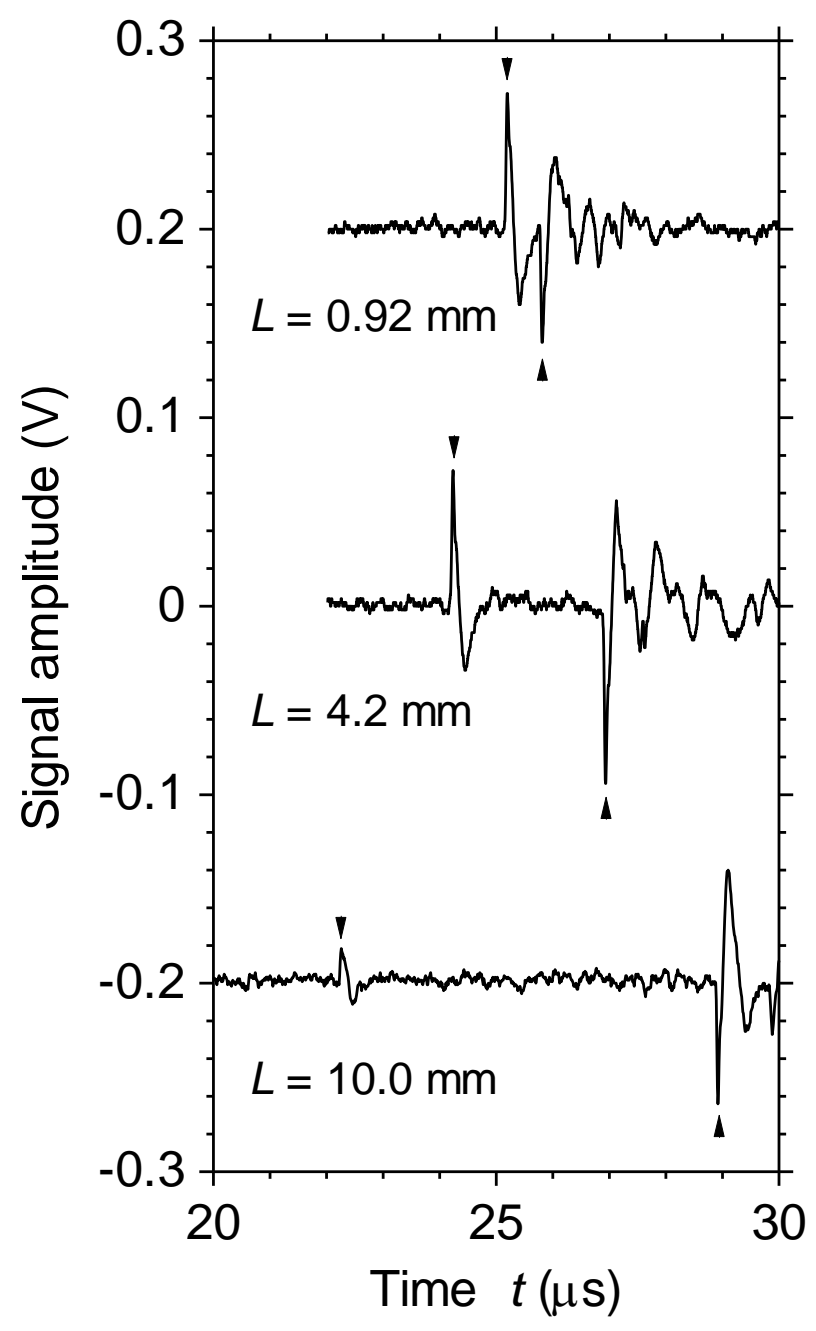

Fig. 3. Signal amplitudes observed from a laser-irradiated aqueous suspension of 8-nm gold nanospheres at $20^{\circ} \mathrm{C}$, as a function of time for different cuvettes: the light pass length of $L=0.92$, 4.2, and $10.0 \mathrm{~mm}$. The gold suspension of the number concentration of $5.70 \times 10^{12}$ spheres/mL was used for these three cuvettes. The signals for $L=0.92$ and $10.0 \mathrm{~mm}$ are shifted from the original data by +0.2 and $-0.2 \mathrm{~V}$, respectively. The arrows indicate two isolated spikes observed.

$1481 \mathrm{~m} / \mathrm{s}$ for water was used. These values are in good agreement with the aforementioned time lags between the first and second peak signals observed in Fig. 3. Therefore, the peaks of the first and second signals in Fig. 3 correspond to the plane waves generated at the inner walls of the cuvette, $Z=L$ and 0 , respectively. Thus, the transducer output was not a pressure pulse signature, but its derivative, 


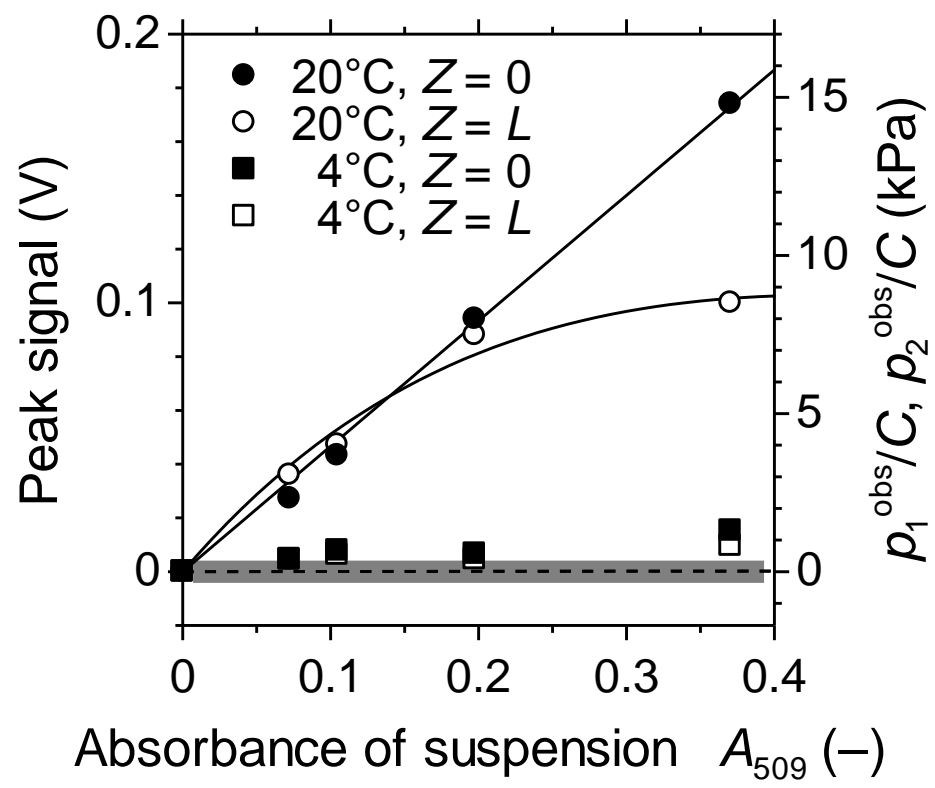

Fig. 4. The peak values of the acoustic signals observed from a laser-irradiated aqueous suspension of 8-nm gold nanospheres in the cuvette of $L=0.92 \mathrm{~mm}$, as a function of the absorbance of the suspension $A_{509}$ for different temperatures of $4{ }^{\circ} \mathrm{C}$ (squares) and $20{ }^{\circ} \mathrm{C}$ (circles). The suspension before enrichment (the number concentration $5.70 \times 10^{12}$ spheres $/ \mathrm{mL}$ ) exhibited $A_{509}=0.0723$. The peak signals are attributable to the acoustic pressures close to the laser-passing inner walls of the cuvette at $Z=0$ (filled symbols) and at $Z=L$ (open symbols). The thick, gray-colored line indicates the level of the background noise. The dashed and solid lines represent the fit of Eqs. (26) and (27) to the experimental data, where $\alpha_{\mathrm{s}}=0.35 \mathrm{~mm}^{-1}$ was used.

profile of photoacoustic signal observed by our experimental setup reflected the shape of a laserirradiated domain of particle suspension in a cuvette rather than the morphology of individual particles.

Henceforth, the results for the cuvettes of $L=0.92 \mathrm{~mm}$ are considered. The peak values of the photoacoustic signals at the different temperatures of 4 and $20{ }^{\circ} \mathrm{C}$ are shown in Fig. 4 , as a function of the observed absorbance of the suspension $A_{509}$. It should be noted that $A_{\lambda}$ is directly proportional to the number concentration of the suspended particles, $n_{\mathrm{p}}$, via Eq. (21), only when the suspensions are sufficiently dilute and the particles are sufficiently small. In the case of $20{ }^{\circ} \mathrm{C}$, the peak value for $Z=0$ 
linearly increased with the absorbance, while that for $Z=L$ linearly increased with the absorbance in the range of $A_{509}<0.2$ and appeared to become maximal or constant in the range of $A_{509}>0.2$. The latter behavior is explained by the effect of laser attenuation. The former behavior is in good agreement with the results of Copland et al. [34], where the photoacoustic signal amplitude from 40-nm gold spheres embedded in the gelatin phantom linearly increases with the concentration up to $n_{\mathrm{p}}=1.2 \times 10^{10}$ spheres/mL. In the case of $4{ }^{\circ} \mathrm{C}$, the peak values were extremely small compared with the case of $20{ }^{\circ} \mathrm{C}$. The peak value for $Z=0$ was somewhat larger than that for $Z=L$ and both peak values slightly increased with the absorbance, although these peak values in the range of $A_{509}<0.2$ were very close to the level of the background noise. Likewise, the photoacoustic signals for an aqueous suspension of 26nm gold spheres irradiated by 5-ns laser pulses disappear at $4{ }^{\circ} \mathrm{C}$ [17].

\subsection{Theoretical estimation of photoacoustic pressure pulses}

Using Eqs. (26) and (27) with Eqs. (21), (23), and (25), we calculated the photoacoustic pressure pulses from a diluted aqueous suspension of $8-\mathrm{nm}$ gold spheres in the cuvette of $L=0.92 \mathrm{~mm}$, which was irradiated by laser pulses $\left[t_{\mathrm{L}}=0.8 \mathrm{~ns}, I_{0}=107 \mu \mathrm{J} /\right.$ pulse, $s_{\mathrm{L}}=0.524 \mathrm{~mm}^{2}, F_{0} \equiv I_{0} / s_{\mathrm{L}}=204 \mathrm{~J} /\left(\mathrm{m}^{2}\right.$ pulse)]. The physical properties of gold and water employed for our calculations are listed in Table 1. As shown in Fig. 4, the theoretical estimations generally agreed with the experimental results. It should be pointed out that $p_{1}^{\text {obs }}$ exhibited a maximum at $A_{509}=\log _{10} e \approx 0.43$. The difference between the peaksignal amplitudes for $Z=0$ and $L$ is attributable mostly to the attenuation of laser beam and partly to the attenuation of generated sound. The laser attenuation did not affect the observed photoacoustic signal for $Z=0$ (i.e., $p_{2}^{\text {obs }}$ ), but affected that for $Z=L$ (i.e., $p_{1}^{\text {obs }}$ ), as expected from Eqs. (27) and (26), respectively. On the other hand, the sound attenuation had an impact on the observed photoacoustic signal for $Z=0$ as compared with that for $Z=L$.

The contributions of the particles and the medium to the acoustic pressure pulse at $20{ }^{\circ} \mathrm{C}$ were estimated as $p_{\mathrm{p}} / p_{\text {tot }}=0.002$ and $p_{\mathrm{m}} / p_{\text {tot }}=0.998$, respectively, where Eq. (25) gave $e_{\mathrm{p}, 0} / e_{\mathrm{tot}, 0}=0.006$ and 
$e_{\mathrm{m}, 0} / e_{\mathrm{tot}, 0}=0.994$ at $t=2 t_{\mathrm{L}}=1.6 \mathrm{~ns}$ (see also Fig. 2b). Thus, the heat generated by the optical power deposition to the 8-nm gold sphere hardly remained therein at the end of laser irradiation, but mostly transferred from the sphere to the surrounding water. This is explained by the large thermal diffusivity of gold: indeed, the distance of thermal diffusion was calculated as $\left(\chi_{\mathrm{p}} t_{\mathrm{L}}\right)^{1 / 2}=319 \mathrm{~nm}$, which was 80fold longer than $r_{\mathrm{p}}=4 \mathrm{~nm}$. Similar results of the heat transfer were obtained at $4{ }^{\circ} \mathrm{C}$. The acoustic pressure pulse at $4{ }^{\circ} \mathrm{C}$ was never contributed by a great amount of the heat within the water medium (i.e., $\left.e_{\mathrm{m}, 0} / e_{\mathrm{tot}, 0}=0.995\right)$ because of $\beta_{\mathrm{water}}=0$ as in Table 1 , but solely by a negligibly small amount of the heat within the $8-\mathrm{nm}$ gold sphere (i.e., $\left.e_{\mathrm{p}, 0} / e_{\mathrm{tot}, 0}=0.005\right)$. This extremely low efficiency of the thermal-toacoustic conversion at $4{ }^{\circ} \mathrm{C}$ resulted in the negligibly small values of $p_{1}^{\text {obs }}$ and $p_{2}^{\text {obs }}$ compared with the case of $20^{\circ} \mathrm{C}$, as in Fig. 4 .

\subsection{Theoretical estimation of temperatures}

Using Eqs. (10) and (11), we computed the temperature profile for the 8-nm gold sphere (the absorption efficiency $Q_{\mathrm{abs}} \equiv \sigma_{\mathrm{abs}} / \pi r_{\mathrm{p}}{ }^{2}=0.311$ and the scattering efficiency $Q_{\text {sca }} \equiv \sigma_{\mathrm{sca}} / \pi r_{\mathrm{p}}{ }^{2}=1.0 \times 10^{-4}$ at $\lambda=509 \mathrm{~nm}$, calculated following Ref. [51]) and the surrounding water at $20{ }^{\circ} \mathrm{C}$, which were irradiated by the laser pulse $\left[t_{\mathrm{L}}=0.8 \mathrm{~ns}\right.$ and $F_{0}=204 \mathrm{~J} /\left(\mathrm{m}^{2}\right.$ pulse $\left.)\right]$. As shown in Fig. 5 , the temperature rise was of the order of $90{ }^{\circ} \mathrm{C}$ during the 0.8 -ns pulsed laser irradiation. The heated volume was highly localized to the vicinity of the sphere, although there was significant heat loss from the sphere to the surrounding water, as in Section 4.2 and Fig. 2b. The temperature of the water medium fell to 1/e of the sphere temperature at a distance of 2.8 and $12.7 \mathrm{~nm}$ from the sphere surface for $t=0.85$ and $1.60 \mathrm{~ns}$, respectively. The latter distance for $t=1.60 \mathrm{~ns}\left(=2 t_{\mathrm{L}}\right.$, immediately after the laser irradiation) corresponds well with the thermal diffusion distance for the laser pulse duration, $\left(\chi_{\mathrm{m}} t_{\mathrm{L}}\right)^{1 / 2}=10 \mathrm{~nm}$, which should be a rough measure for the thickness of the high-temperature region of water around the heated gold sphere (i.e., the near-field medium explained in Section 3.1). In the case of $4{ }^{\circ} \mathrm{C}$, we 


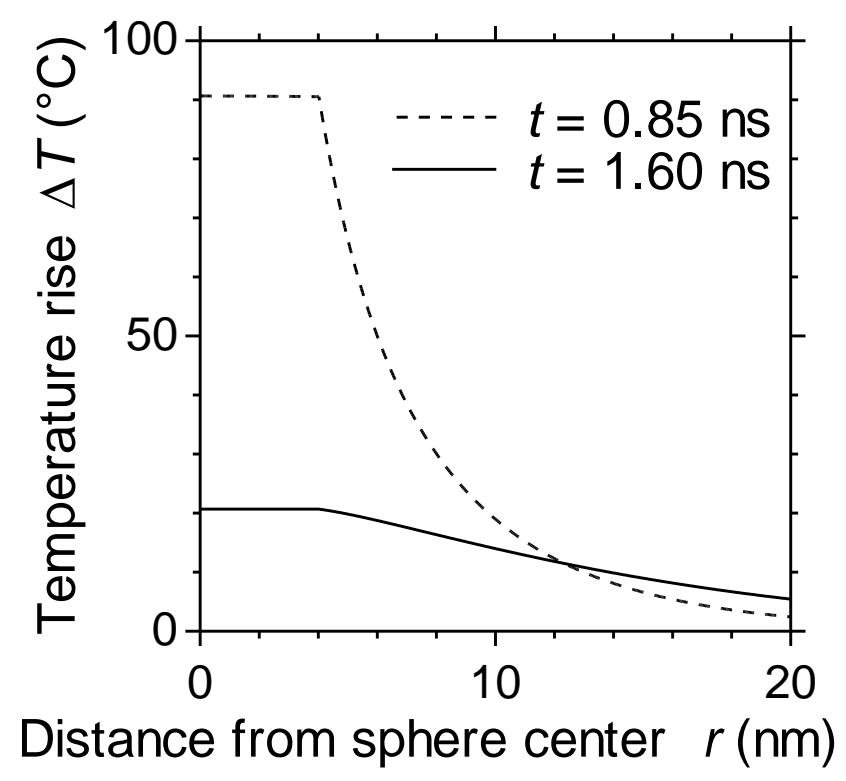

Fig. 5. The spatial distribution of the temperature rise, $\Delta T(r, t)$, around a 8-nm gold nanosphere at $20{ }^{\circ} \mathrm{C}$ in the absence of laser attenuation. The solid and the dashed lines correspond to the distributions at $t=1.60 \mathrm{~ns}\left(=2 t_{\mathrm{L}}\right.$, immediately after the laser irradiation) and $t=0.85 \mathrm{~ns}$ (the time when the temperature of the gold nanosphere became maximal), respectively.

obtained almost the same results as in Figs. $2 \mathrm{~b}$ and 5 for $20{ }^{\circ} \mathrm{C}$. Thus, the suspension of the gold nanospheres in water irradiated by the nanosecond laser pulses exhibited almost the same behavior of the heat production/transfer at different temperatures of 4 and $20^{\circ} \mathrm{C}$, although the acoustic responses at these two temperatures were different from each other as in Fig. 4.

\section{Concluding remarks}

We theoretically described the acoustic pressure pulse from the suspensions of metal nanospheres in the case of low-level laser irradiation. We also carried out the experimental measurements of the photoacoustic signals, where the suspensions of 8-nm gold nanospheres in water at different 
concentrations and different temperatures were irradiated by 0.8 -ns laser pulses. The photoacoustic signals at $4{ }^{\circ} \mathrm{C}$ were found to be negligibly smaller than those at $20^{\circ} \mathrm{C}$. Our physical model suggested that (i) the heat generated by the optical power deposition to the 8-nm gold sphere hardly remained therein, but mostly transferred from the sphere to the surrounding water at both 4 and $20{ }^{\circ} \mathrm{C}$, and that (ii) the acoustic pressure at $4{ }^{\circ} \mathrm{C}$ was never contributed by a great amount of the heat within the water medium because of $\beta_{\text {water }}$ (thermal coefficient of volume expansion for water) $=0$, but solely by a negligibly small amount of the heat within the 8-nm gold sphere. This extremely low efficiency of the thermal-to-acoustic conversion at $4{ }^{\circ} \mathrm{C}$ explained the negligibly small photoacoustic signals compared with the case of $20^{\circ} \mathrm{C}$.

\section{Acknowledgements}

This work was partly supported by the Innovative Techno-Hub for Integrated Medical Bio-imaging of the Project for Developing Innovation Systems, from the Ministry of Education, Culture, Sports, Science and Technology (MEXT), Japan. 


\section{References}

[1] D. Cahen, G. Bults, H. Garty, S. Malkin, J. Biochem. Biophys. Methods, 3 (1980) 293-310.

[2] C. Kim, C. Favazza, L.H.V. Wang, Chem. Rev., 110 (2010) 2756-2782.

[3] S. Mallidi, G.P. Luke, S. Emelianov, Trends Biotechnol., 29 (2011) 213-221.

[4] X. Yang, E.W. Stein, S. Ashkenazi, L.V. Wang, WIREs Nanomed. Nanobiotechnol., 1 (2009) 360368.

[5] A.A. Oraevsky, in: L.V. Wang (Ed.) Photoacoustic Imaging and Spectroscopy, CRC Press, Boca Raton, 2009, Chapter 30.

[6] G. Luke, D. Yeager, S. Emelianov, Ann. Biomed. Eng., 40 (2012) 422-437.

[7] S. Mallidi, T. Larson, J. Tam, P.P. Joshi, A. Karpiouk, K. Sokolov, S. Emelianov, Nano Lett., 9 (2009) $2825-2831$.

[8] S. Kumar, J. Aaron, K. Sokolov, Nat. Protoc., 3 (2008) 314-320.

[9] X.H. Huang, P.K. Jain, I.H. El-Sayed, M.A. El-Sayed, Laser Med. Sci., 23 (2008) 217-228.

[10] V.P. Zharov, K.E. Mercer, E.N. Galitovskaya, M.S. Smeltzer, Biophys. J., 90 (2006) 619-627.

[11] C.M. Pitsillides, E.K. Joe, X.B. Wei, R.R. Anderson, C.P. Lin, Biophys. J., 84 (2003) 4023-4032.

[12] P.C. Li, C.R.C. Wang, D.B. Shieh, C.W. Wei, C.K. Liao, C. Poe, S. Jhan, A.A. Ding, Y.N. Wu, Opt. Express, 16 (2008) 18605-18615.

[13] A. Agarwal, S.W. Huang, M. O'Donnell, K.C. Day, M. Day, N. Kotov, S. Ashkenazi, J. Appl. Phys., 102 (2007) 064701.

[14] C. Kim, E.C. Cho, J.Y. Chen, K.H. Song, L. Au, C. Favazza, Q.A. Zhang, C.M. Cobley, F. Gao, Y.N. Xia, L.H.V. Wang, ACS Nano, 4 (2010) 4559-4564.

[15] S.E. Skrabalak, J. Chen, L. Au, X. Lu, X. Li, Y.N. Xia, Adv. Mater., 19 (2007) 3177-3184.

[16] C. Kim, H.-M. Song, X. Cai, J. Yao, A. Wei, L.V. Wang, J. Mater. Chem., 21 (2011) 2841.

[17] Y.-S. Chen, W. Frey, S. Aglyamov, S. Emelianov, Small, 8 (2012) 47-52.

[18] Y.-S. Chen, W. Frey, S. Kim, P. Kruizinga, K. Homan, S. Emelianov, Nano Lett., 11 (2011) 348354. 
[19] Y.-S. Chen, W. Frey, S. Kim, K. Homan, P. Kruizinga, K. Sokolov, S. Emelianov, Opt. Express, 18 (2010) 8867-8877.

[20] C. Loo, A. Lowery, N.J. Halas, J. West, R. Drezek, Nano Lett., 5 (2005) 709-711.

[21] Y.W. Wang, X.Y. Xie, X.D. Wang, G. Ku, K.L. Gill, D.P. O'Neal, G. Stoica, L.V. Wang, Nano Lett., 4 (2004) 1689-1692.

[22] M. Pramanik, K.H. Song, M. Swierczewska, D. Green, B. Sitharaman, L.H.V. Wang, Phys. Med. Biol., 54 (2009) 3291-3301.

[23] A. De La Zerda, C. Zavaleta, S. Keren, S. Vaithilingam, S. Bodapati, Z. Liu, J. Levi, B.R. Smith, T.J. Ma, O. Oralkan, Z. Cheng, X.Y. Chen, H.J. Dai, B.T. Khuri-Yakub, S.S. Gambhir, Nat. Nanotechnol., 3 (2008) 557-562.

[24] Z. Liu, W.B. Cai, L.N. He, N. Nakayama, K. Chen, X.M. Sun, X.Y. Chen, H.J. Dai, Nat. Nanotechnol., 2 (2007) 47-52.

[25] J.W. Kim, E.I. Galanzha, E.V. Shashkov, H.M. Moon, V.P. Zharov, Nat. Nanotechnol., 4 (2009) 688-694.

[26] J. Yu, D. Javier, M.A. Yaseen, N. Nitin, R. Richards-Kortum, B. Anvari, M.S. Wong, J. Am. Chem. Soc., 132 (2010) 1929-1938.

[27] G. Kim, S.W. Huang, K.C. Day, M. O'Donnell, R.R. Agayan, M.A. Day, R. Kopelman, S. Ashkenazi, J. Biomed. Opt., 12 (2007) 044020.

[28] K. Wilson, K. Homan, S. Emelianov, Nat. Commun., 3 (2012) 618.

[29] J.R. McLaughlan, R.A. Roy, H.Y. Ju, T.W. Murray, Opt. Lett., 35 (2010) 2127-2129.

[30] P. Sharma, S.C. Brown, N. Bengtsson, Q.Z. Zhang, G.A. Walter, S.R. Grobmyer, S. Santra, H.B. Jiang, E.W. Scott, B.M. Moudgil, Chem. Mater., 20 (2008) 6087-6094.

[31] A.A. Oraevsky, A.A. Karabutov, E.V. Savateeva, Proc. SPIE, 4434 (2001) 60-69.

[32] S. Egerev, S. Ermilov, O. Ovchinnikov, A. Fokin, D. Guzatov, V. Klimov, A. Kanavin, A. Oraevsky, Appl. Opt., 48 (2009) C38-C45.

[33] A.L. McKenzie, Phys. Med. Biol., 35 (1990) 1175-1209. 
[34] J.A. Copland, M. Eghtedari, V.L. Popov, N. Kotov, N. Mamedova, M. Motamedi, A.A. Oraevsky, Mole. Imaging Biol., 6 (2004) 341-349.

[35] O.M. Zozulya, O.V. Puchenkov, Acoust. Phys., 39 (1993) 46-50.

[36] V.N. Inkov, A.A. Karabutov, I.M. Pelivanov, Laser Phys., 11 (2001) 1283-1291.

[37] Y. Cao, G.J. Diebold, Opt. Eng., 36 (1997) 417-422.

[38] S.S. Alimpiev, Y.O. Simanovskii, S.V. Egerev, A.E. Pashin, Laser Chem., 16 (1995) 63-73.

[39] C.P. Lin, M.W. Kelly, Appl. Phys. Lett., 72 (1998) 2800-2802.

[40] A. Conjusteau, S.A. Ermilov, D. Lapotko, H. Liao, J. Hafner, M. Eghtedari, M. Motamedi, N. Kotov, A.A. Oraevsky, Proc. SPIE, 6086 (2006) 60860K.

[41] M.G. Gonzalez, X. Liu, R. Niessner, C. Haisch, Appl. Phys. Lett., 96 (2010) 174104-174103.

[42] M. Kitz, S. Preisser, A. Wetterwald, M. Jaeger, G.N. Thalmann, M. Frenz, Biomed. Opt. Express, 2 (2011) 291-304.

[43] S. Egerev, O. Ovchinnikov, A. Fokin, Acoust. Phys., 51 (2005) 160-166.

[44] S.V. Egerev, A.A. Oraevsky, Int. J. Thermophys., 29 (2008) 2116-2125.

[45] V. Zharov, D. Lapotko, Rev. Sci. Instrum., 74 (2003) 785-788.

[46] V. Kotaidis, A. Plech, Appl. Phys. Lett., 87 (2005) 213102-213103.

[47] S.R. Aglyamov, A.B. Karpiouk, F. Bourgeois, A. Ben-Yakar, S.Y. Emelianov, Opt. Lett., 33 (2008) 1357-1359.

[48] E.-A. Brujan, Microfluid. Nanofluid., 11 (2011) 511-517.

[49] L.V. Wang, IEEE J. Sel. Top. Quant. Electron., 14 (2008) 171-179.

[50] A.S. Dukhin, P.J. Goetz, Characterization of Liquids, Nano- and Microparticulates, and Porous Bodies using Ultrasound, 2nd edn., Elsevier, Amsterdam, 2010.

[51] P.K. Jain, K.S. Lee, I.H. El-Sayed, M.A. El-Sayed, J. Phys. Chem. B, 110 (2006) 7238-7248. 
Table 1 Physical properties of gold (particle) and water (medium) at ambient conditions.

\begin{tabular}{|c|c|c|c|}
\hline Physical property & Gold & $\begin{array}{l}\text { Water } \\
\text { at } 4{ }^{\circ} \mathrm{C}\end{array}$ & $\begin{array}{c}\text { Water } \\
\text { at } 20^{\circ} \mathrm{C}\end{array}$ \\
\hline$\rho_{\mathrm{i}}:$ Mass density $\left(\mathrm{kg} \mathrm{m}^{-3}\right)$ & 19320 & 1000 & 998 \\
\hline$c_{\mathrm{i}}:$ Specific heat capacity $\left(\mathrm{J} \mathrm{kg}^{-1} \mathrm{~K}^{-1}\right)$ & 128 & 4205 & 4182 \\
\hline$k_{\mathrm{i}}$ : Thermal conductivity $\left(\mathrm{J} \mathrm{K}^{-1} \mathrm{~m}^{-1} \mathrm{~s}^{-1}\right)$ & $(315)^{\mathrm{a}}$ & 0.567 & 0.599 \\
\hline$\chi_{\mathrm{i}}:$ Thermal diffusivity $\left(\mathrm{m}^{2} \mathrm{~s}^{-1}\right)$ & $\left(1.27 \times 10^{-4}\right)^{\mathrm{a}}$ & $1.35 \times 10^{-7}$ & $1.43 \times 10^{-7}$ \\
\hline$\beta_{\mathrm{i}}:$ Thermal coefficient of volume expansion $\left(\mathrm{K}^{-1}\right)$ & $4.27 \times 10^{-5}$ & 0 & $2.06 \times 10^{-4}$ \\
\hline$\kappa_{\mathrm{i}}:$ Isothermal compressibility $\left(\mathrm{Pa}^{-1}\right)$ & $5.88 \times 10^{-12}$ & $4.96 \times 10^{-10}$ & $4.59 \times 10^{-10}$ \\
\hline$\Gamma_{\mathrm{i}}=\beta_{\mathrm{i}} / \kappa_{\mathrm{i}} c_{\mathrm{i}} \rho_{\mathrm{i}}:$ Grüneisen parameter $(-)$ & 2.94 & 0 & 0.108 \\
\hline Dimensionless prefactor for $p_{\mathrm{i}}$ in Eqs. (6) and (23) & $0.038^{\mathrm{b}}$ & $0^{c}$ & $0.108^{c}$ \\
\hline
\end{tabular}

\footnotetext{
${ }^{\mathrm{a}}$ The value in the parenthesis was not used in the solutions of the heat transfer equations.

${ }^{\mathrm{b}}$ The value of the prefactor $\kappa_{\mathrm{p}} \Gamma_{\mathrm{p}} / \kappa_{\mathrm{m}}$ for the particle contribution in Eqs. (6) and (23).

${ }^{c}$ The value of the prefactor $\Gamma_{\mathrm{m}}$ for the medium contribution in Eqs. (6) and (23).
} 